http://jmscr.igmpublication.org/home/

ISSN (e)-2347-176x ISSN (p) 2455-0450

crossref DOI: https://dx.doi.org/10.18535/jmscr/v7i8.147

Journal Of Medical Science And Clinical Research

IGM Publication

An Official Publication of IGM Publication

\title{
A Comparative Study of Icthammol Glycerine with Antibiotic Ear Drops in Furunculosis
}

\author{
Authors \\ Dr Harshvardhan ${ }^{1}$, Dr Surbhi ${ }^{2}$ \\ ${ }^{1}$ Senior Resident, Patna Medical College and Hospital \\ ${ }^{2}$ Junior Resident 3, Patna Medical College and Hospital, Patna, India
}

\begin{abstract}
Furunculosis is a localised form of infection of sole hair follicle. Since hair follicle is present only in the cartilaginous segment therefore furuncle is seen only on the lateral segment of external auditory canal. Staphylococcus is the chief infective organism. Diagnosis is mainly clinically by presenting symptoms and otoscopic examination. Topic antibiotics have tried but with varied results.

Material \& Methods: This is a randomized clinical trial study done in our ENT OPD at Patna medical college, Patna. After proper examination and consent.

Objective: To compare the efficacy of icthmmol glycerine and antibiotic ear drops active against staphylococcus aureus in treatment of furunculosis of ear.

Results: Though the results of both the treatments were alike at the end of treatment period, symptoms improvement with icthammol glycerine was much quicker and before than antibiotic ear drops.

Keywords: Furuncle, Furunculosis, Antibiotic, Icthmmol Glycerine.
\end{abstract}

\section{Introduction}

Furunculosis is localized otitis externa. It is caused by staphylococcal infection of a single hair follicle. Oral antibiotics are the treatment of choice before abscess formation. Incision and drainage are recommended if an abscess forms. Severe associated soft-tissue infection or cellulitis is an indication for systemic antibiotic therapy. The clinical efficacy of glycerine-ichthammol in otitis externa may be due to an anti-inflammatory action of ichthammol or a dehydrating effect of glycerine on the oedematous ear canal. A study of the antibacterial property of glycerine-ichthammol showed inhibition of selected gram positive organisms (Streptococcus pyogenes and Staphylococcus aureus) by ichthammol and glycerine-ichthammol combination, but only negligible antibacterial activity against Pseudomonas aeruginosa and Escherichia coli. Candida albicans was also weakly inhibited.

\section{Material and Methods}

This is randomized clinical trial which was conducted in department of otolaryngology, Patna medical college over a period of one year from December 2017 to November 2018. Patients presenting in the ENT outpatient department were examined and were elected for study purpose after written consent. Case was confirmed after complete history followed by otoscopic examination of ear. Dry mopping was done in required cases. Main presenting symptoms for 
diagnosis were ear pain, ear heaviness and discharge.

\section{Inclusion Criteria}

1) Age group between 12 to 50

2) Clinically confirmed cases of furuncolosis with other disease excluded

3) Those giving consent for study and regular follow up

4) Localised infection

\section{Exclusion Criteria}

1) Age less than 12 years and more than 50 years.

2) History of chronic otitis media.

3) Uncontrolled dibetes mellitus .

4) Those no giving consent for study upon them

Patients were randomly allocated between group 1 and group 2 of 50 patient each. Group 1 patient was provided with icthammol glycerine ear wick which was changed every day and group 2 was given antibiotic ear drops. Each patient was given an oral antibiotic coverage with amoxicillin group for one week. Trial subject was evaluated on visual analog scale based on symptoms i.e ear pain, ear heaviness and discharge. Ear was examined on all the follow up days 3,5, and 7.After all the patients were completed with their follow up of 2 week, result was analysed. Results were checked and compared to adjust $\mathrm{p}$ values.

\section{Results}

Total number of patients which were examined in this study was 100 .Fifty patients were randomly allotted in each group. Symptoms in both the group was noted at the start of study. Common symptoms in both the groups were were asked from each patient in each group. Groups which was divided was group 1 and group 2.Group 2 was given antibiotic ear drops and group 1 patients ear was packed with icthammol glycerine $10 \% 24$ hourly. Both the groups patients were followed up on $3^{\text {rd }}, 5^{\text {th }}$ and $7^{\text {th }}$ day and symptoms was recorded from each patient followed by otoscopic examination of ear canal ,if required dry mopping of the canal was done.fig 1

\begin{tabular}{|l|c|c|}
\hline & GROUP 1 & GROUP 2 \\
\hline PAIN & 48 & 49 \\
\hline HEAVINESS & 50 & 50 \\
\hline DISCHARGE & 46 & 48 \\
\hline
\end{tabular}

\section{Fig 1}

On day 1 both the groups patients had equal symptoms. When compared to symptom on day 1 to day 3, change was significant $(\mathrm{P}<.05)$. Statistically significant difference was seen in both the groups. Group 1 showed more improvement in pain and heaviness than group 2 . Discharge has also decreased more in group 1 compared to group 2.fig 2

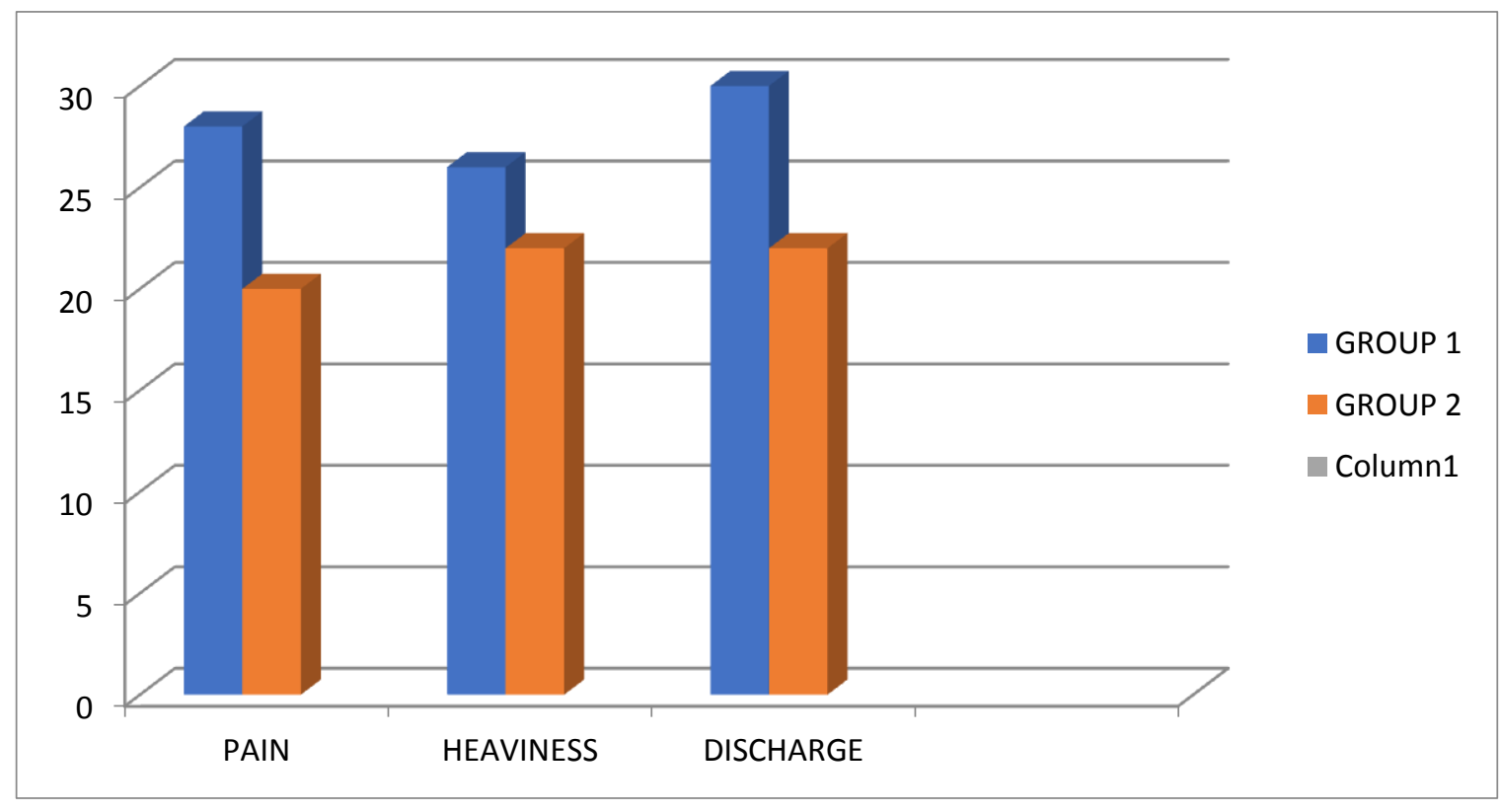

Fig 2 
When examined on day $5^{\text {th }}$, both the group showed much more improvement in symptoms. Group 1 showed almost absolute resolution of most of the symptoms. Discharge was scanty in most of the patients. Symptoms of pain and heaviness in both the groups was comparable and showed diminish in both the groups .However number of patients with improved symptoms were more in group 1.fig 3

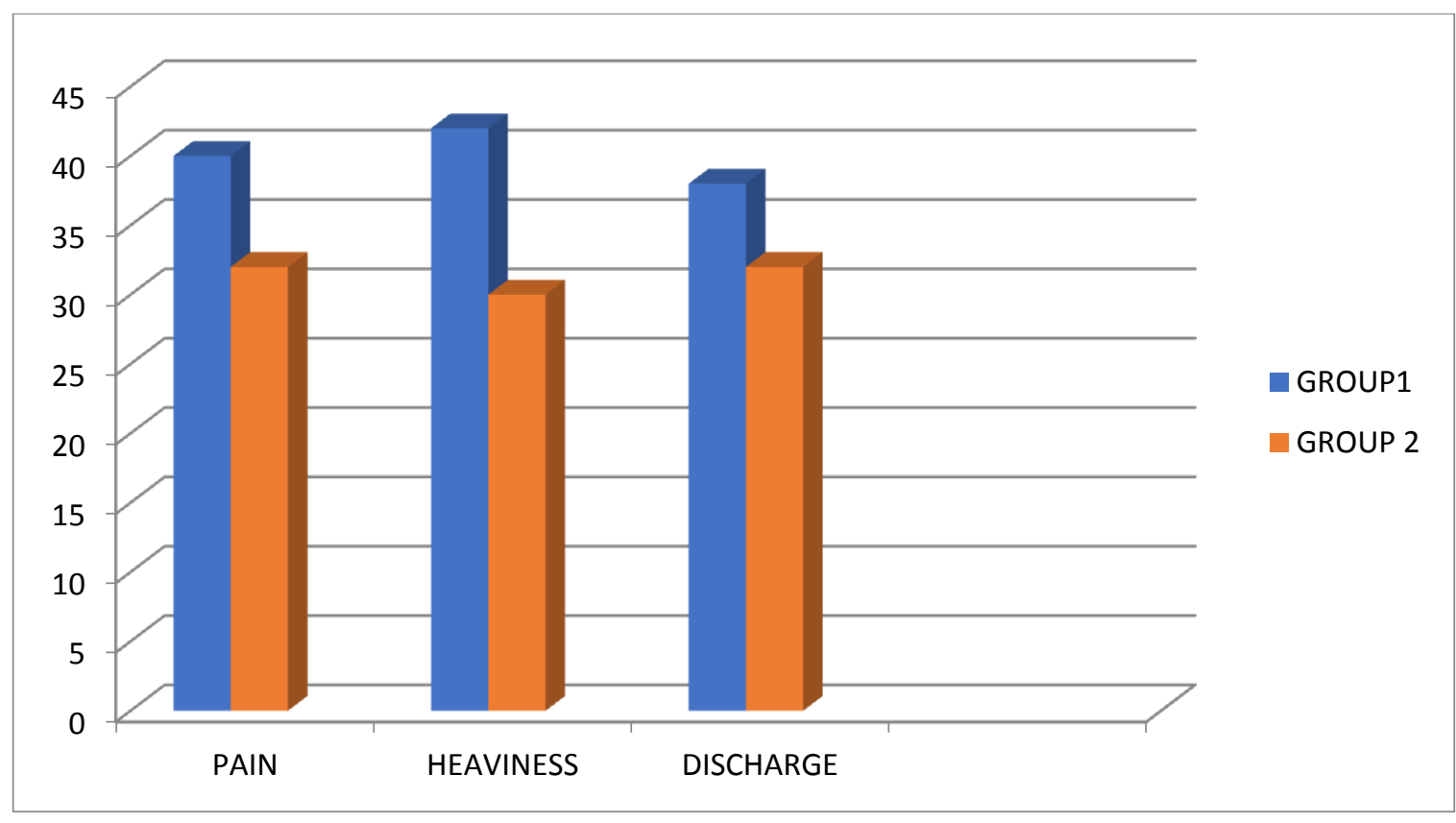

Follow up on day $7^{\text {th }}$, results were akin. Group 1

Fig 3 and group 2 both had recovered in their pain, heaviness and discharge symptoms. There was complete resolution of symptoms in both the groups in maximum number of patient. Although the number of patient with complete resolution of

symptoms was more in iccthamol glycerine group. fig 4

Canal edema was seen to turn down soon with icthmmol glycerine wick but was later on with the use of drops.

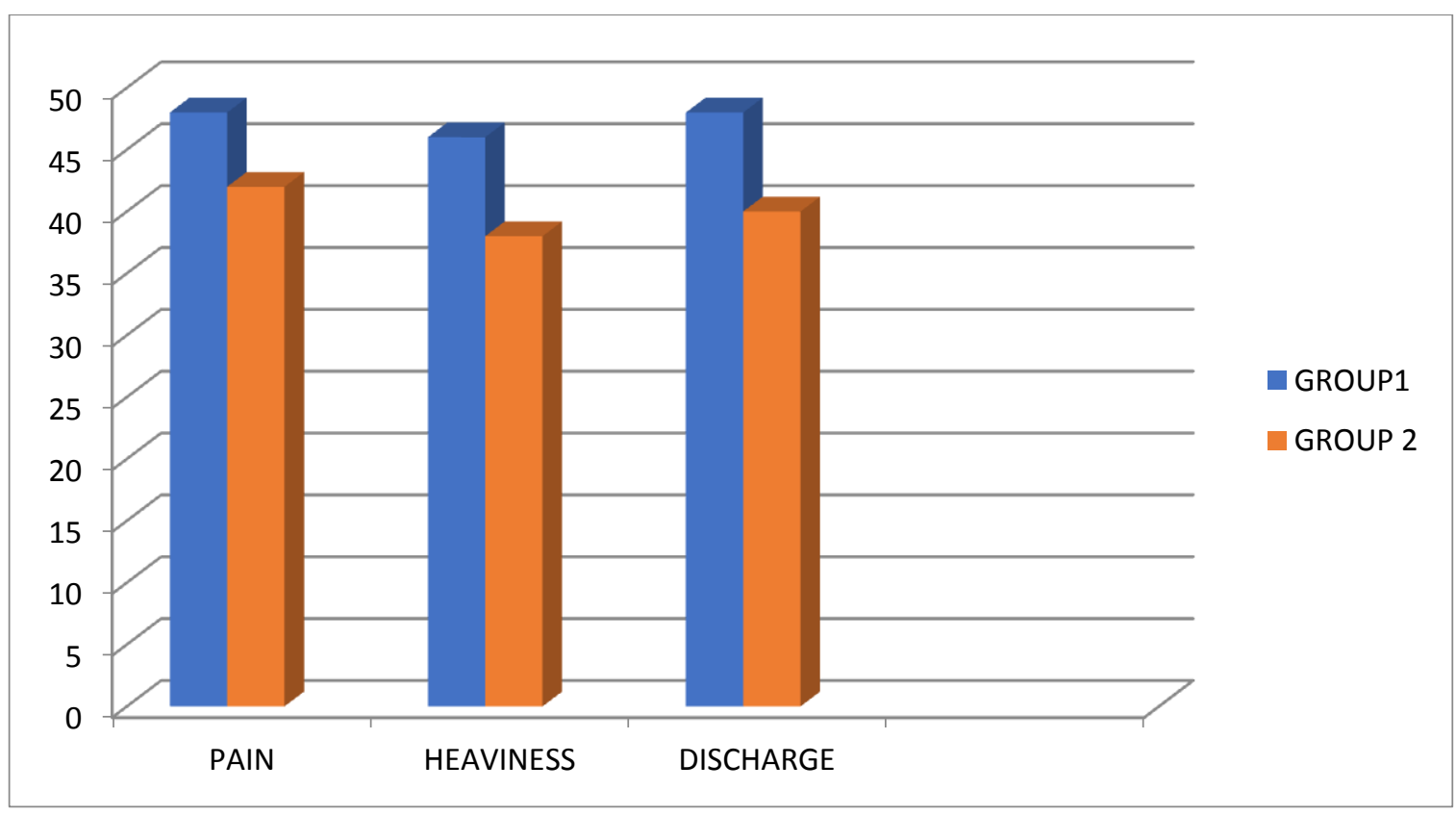

Fig 4 


\section{Discussion}

Furunculosis is a local form of otitis externa and is a infection of single hair follicle. Hair follicle being present only on the outer part of external auditory canal, therefore a furuncle happens to occour only on the outer part of canal which is cartilaginous part. Bacteria invades hair follicle and leads to well confined deep skin infection. Further progression leads to pustule, abscess and may be associated with cellulitis and edema. Bacteria multiplies inside the ostium of hair follicle ${ }^{1}$. Key presenting symptom is pain, patient often feels heaviness in the ear with scanty serous discharge. Tragal tenderness is mostly present. Otoscopic examination usually establishes the diagnosis. Staphylococcus aureus (S. aureus) is the most widespread organism causing furunculosis. The $\mathrm{S}$. aureus frequently express genes for Panton-Valentine leucocidin $(\mathrm{PVL})^{2}$ Leucocidal toxins isolated from pathogenic strains of S. aureus trigger lysis of phagocytic cells and may have an central role in cutaneous infection ${ }^{3 .}$ Sporadic cases of furunculosis happen when pathogenic organisms are introduced into the canal in the circumstance of other local risk factors .Several conditions emerge to be associated with recurrent furunculosis including hypogammaglobulinaemia, diabetes mellitus and dysphagocytosis ${ }^{4,5}$.

If untreated, the infection usually progresses to a localized abscess, which then discharges into the external ear canal. Providing there is enough drainage, the infection will resolve of your own accord. The infection can also extend towards the deeper tissues, where it may cause a diffuse softtissue infection spreading to the pinna, postauricular skin and parotid gland. Recurrent infection can cause enduring scarring and fibrosis of the external canal with following meatal stenosis. Eventually, this may also dispose to chronic disseminated otitis externa.

Furunculosis of the external canal is exquisitely painful and suitable analgesics should be obtainable to all patients. No data exists that compares the efficacy of different analgesics. A recent systematic review of treatment for otitis externa specifically excluded furunculosis from consideration. ${ }^{6} \quad$ Oral or systemic antistaphylococcal antibiotics like penicillinaseresistant penicillin, macrolide, cephalosporin, clindamycin or quinolone and topical treatment (antibiotics, astringents, hygroscopic dehydrating agents) are used for treatment.

Oral antibiotic treatment is recommended in the early stages of the disease. Severe spreading softtissue infection should be treated with intravenous antibiotic therapy. Incision and drainage is advocated if abscess is formed. Topical treatment is preferable, if abscess has discharged, surgically or spontaneously. Topical antibiotics active against staphylococcus are usually prescribed. Placing a wick into the ear canal facilitates treatment in the presence of severe canal oedema and narrowing. Glycerol and ichthammol solution has a precise antistaphylococcal action ${ }^{7,8}$ and is hygroscopic, thus causing dehydration of the canal tissue. Aluminium acetate solution is an astringent as well as a hygroscopic agent. Other choices are eradication therapy with nasal mupirocin or with oral flucloxacillin or azithromycin for 14 days. Bacterial intervention therapy which is intentionally implanting a non-pathogenic strain of S. aureus (strain 502A is the most popular) to recolonize the nares and skin.

It has been reported that correction of specific biochemical abnormalities (e.g. hypoferraemia, low serum zinc) may lead to a marked drop in the incidence of infections.

\section{Conclusion}

Furunculosis is a localised form of infection of single hair follicle. Oral antibiotic treatment is suggested in the early stages of the disease. Topical treatment is preferable. Topical antibiotics active against staphylococcus are usually prescribed. Insertion of a wick into the ear canal facilitates treatment in the presence of severe canal oedema and narrowing. Glycerol and ichthammol solution has a specific antistaphylococcal action and is hygroscopic, thus 
causing dehydration of the canal tissue. The result of using topical antibiotic ear drop is comparable to icthammol and glycerine but signs and symptoms improve faster and earlier with glycerine and icthmmol than with topic antibiotics.

\section{References}

1. Abe Y, Akiyama H, Arata J. Furunclelike lesions in mouse experimental skin infections with Staphylococcus aureus. J Dermatol 1993; 20: 198-202.

2. Durupt F, Mayor L, Bes M, et al. Prevalence of Staphylococcus aureus toxins and nasal carriage in furuncles and impetigo. Br J Dermatol 2007; 157: 11617.

3. Szmigielski S, Prevost G, Monteil H, et al. Leukocidal toxins of staphylococci. Zentralbl Bakteriol 1999; 289: 185-201.

4. furunculosis: Bacteriological findings and epidemiology in 100 cases. Scand J Infect Dis 1981; 13: 115-19.

5. Wertheim HF, Melles DC, Vos MC, et al. The role of nasal carriage in Staphylococcus aureus infections. Lancet Infect Dis 2005; 12: 751-62.

6. Kaushik V, Malik T, Saeed SR. Interventions for acute otitis externa. Cochrane Database Syst Rev 2010; 20: CD004740. 10

7. Ahmed K, Roberts ML, Mannion PT. Antimicrobial activity of glycerineichthammol in otitis externa. Clin Otolaryngol Allied Sci 1995; 20: 201-3.

8. Nilssen E, Wormald PJ, Oliver S. Glycerol and ichthammol: medicinal solution or mythical potion? J Laryngol Otol 1996; 110: 319-21. 